Research Article

\title{
Comparison of subjective and functional results on the operative and non-operative application of clavicle fractures
}

Haris Dwi Khoirur Rofiq ${ }^{1 *}$, Erwin Ramawan ${ }^{1}$

1) Department of Orthopaedic and Traumatology, Faculty of Medicine Universitas Airlangga- Dr.Soetomo General Hospital, Surabaya

A R T I C L E I N F O

Submitted : $26^{\text {th }}$ February 2022

Accepted : $2^{\text {nd }}$ August 2021

Published : 25 $5^{\text {th }}$ January 2021

Keywords:

Clavicle fracture, operative treatment, DASH score, SF-36 score

\section{*Correspondence:}

dr.harisdwi@gmail.com

This is an Open acces article under the CC-BY license

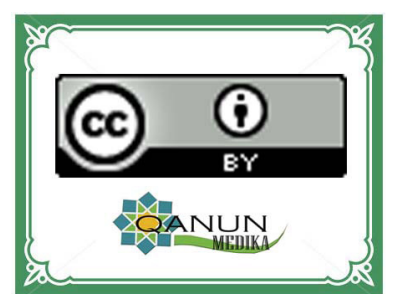

\section{ABSTRACT}

A clavicle fracture is one of the most common bone injuries occurring in 2-5\% of all adult fractures. Clavicle fractures rarely require surgery. Most of the cases were treated by conservative methods. Conservative treatment yields satisfactory results. However, some latest studies showed an incidence of nonunion of up to $15 \%$ and patient dissatisfaction of $31 \%$. This study analyzed the outcome and incidence of complications between operative therapy compared to conservative treatment. This research design was a retrospective descriptive observative. The study population was all patients diagnosed with clavicle fracture of the middle third in RSUD Dr. Soetomo Surabaya from 2015 to 2019. The total sample was 153 patients, with 95 patients who underwent conservative measures and 58 patients who underwent surgery. From the results, there were significant differences between the mean SF-36 score [90.81 and $98.21(\mathrm{p}=0.000)$ ], the mean DASH score [6.94 and $2.42(\mathrm{p}=0.000)]$ in patients with fractures clavicle middle third treated with conservative and operative treatment. The union and functional rates were also better in the operative group. This study shows that the operative treatment on clavicle fractures is superior compared to conservative therapy. 


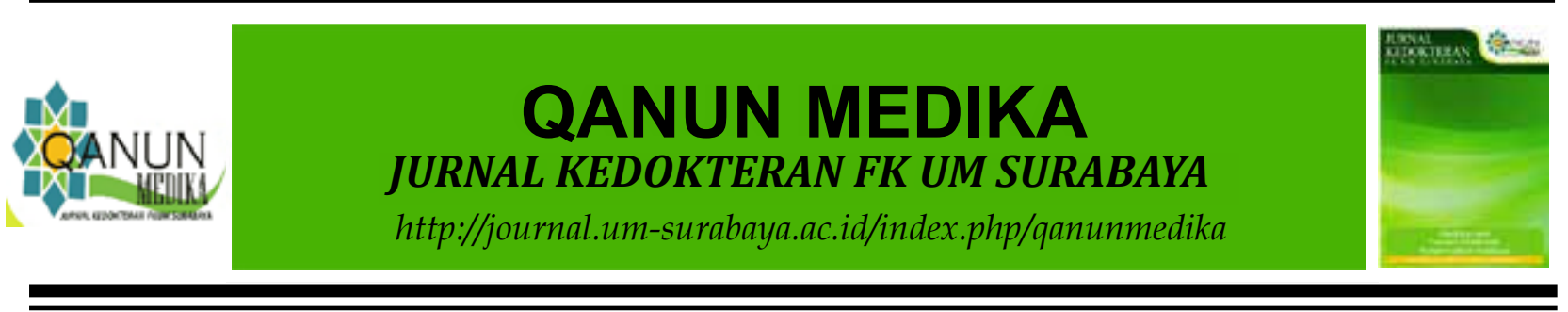

\section{INTRODUCTION}

A clavicle fracture is one of the bone injuries that occurs most frequently in $2-5 \%$ of all adult fractures, with an incidence of 29-64 cases per 100,000 (Robinson, 1998). Younger individuals often experience these injuries due to high-energy trauma, such as motor vehicle accidents or sports injuries. In contrast, older individuals are more likely to experience injuries due to low-energy trauma such as slips and falls at home (Kihlström et al., 2017).

Clavicle fractures rarely require surgery. Mostly, the treatment was conservative. Most cases of middle third clavicle fractures can be treated with various immobilization methods. Thus conservative therapy is the primary modality in managing these fractures (Naveen, Joshi, and Harikrishnan, 2017). Conservative treatment yields satisfactory results with a high union rate and a relatively low nonunion rate of $0.03-6.2 \%$. However, recent research shows that nonunion incidence is up to $15 \%$, and patient dissatisfaction is $31 \%$ ( $\mathrm{Jha}$ et al., 2018). Conservative treatment can also cause functional disorders of the shoulder and lumps, which are cosmetically unfavorable. Operative management maintaining standard bone length and alignment can prevent the disadvantages of conservative treatment. Good outcomes with high union rates and low complication rates have been achieved with operative modalities. However, operative treatment is not without weaknesses; operative treatment can lead to surgical wound infections, hypertrophic scars, protruding implants, and re-surgery when removing the implants (Naveen et al., 2017).

Orthopedic surgeons still have doubts about the gold standard to treat clavicle fractures, whether operative or conservative therapy. This study analyzed the outcome and the incidence of complications from malunion and nonunion between operative therapy using a plate and screw compared to conservative treatment.

\section{METHODS}

This research design was a retrospective descriptive observative. The study population was all patients diagnosed with closed fracture middle third of clavicle in RSUD Dr. Soetomo Surabaya from 2015 to 2019. This study's sample was all patients who met the inclusion criteria. The inclusion criteria were patients with a history of closed fracture in the middle third of the clavicle, both male and female, who have surgery and are treated conservatively at Dr. Soetomo, and were willing to be evaluated. Data were collected at the orthopedic and traumatology polyclinic Dr. Soetomo hospital. Exclusion criteria in this study were patients with a history of fractures other than clavicle fractures, multi-trauma (injury to more than 1 region of the body), communication problems, and refusal to participate in the study. The evaluation time of both groups was one year after the time of the incident. Conservative was mean that the patient treated using arm sling or ransel verband. The operative was mean that the patient underwent operation with plate and screw fixation.

Disability of Arm, Shoulder, and Hand (DASH) questionnaire score is an instrument with specific results that are managed and developed as a measuring tool for disability and upper extremity disorders that we can assess for ourselves. DASH consists of 30 items of scoring points, a score of 0 (without disability) to 100 (Gummesson, Atroshi, and Ekdahl, 2003)

The Medical Outcomes Study Short-Form Health Survey (SF-36) is a health status questionnaire developed in two decades to assess patients' functional status and health. SF36 has been used for various clinical purposes, 
including orthopedics and traumatology (Zhang et al., 2015). The SF-36 is a product of The Medical Outcomes Study (MOS), a four-year study examining specific medical treatment outcomes. Starting from the 149item Functional and Well Being Profile used by MOS, 20 short survey items named SF20 were formed in collaboration with RAND Corporation published SF-36 in 1992 (Laucis et al., 2015). SF-36 is a questionnaire containing 36 question items that evaluate the physical function, social function, activity limitations due to physical \& mental health, vitality, pain, and general health status (Carreon et al., 2011).

Data of patients of closed fracture middle third of clavicle both have undergone surgery and conservative treatment about subjective and functional outcome data in gender, age, site of injury, dominant hand, DASH Score, SF-36 retrieved from medical records. The collected data were analyzed with SPSS using a nonparametric independent sample test.

\section{RESULTS}

There were 531 cases of patients with closed fracture middle third of the clavicle from the database search. The patient with multitrauma was 182 patients, multiple fractures were 96 patients, and loss of contact was 100 patients. The final data that match the criteria obtained data of 153 patients with clavicle fracture middle third who came to Dr. Soetomo Hospital period January 2015 to January 2019. From the data obtained, 95 patients performed conservative actions, and 58 patients performed operative actions.
Characteristics found that the male sex was 110 people $(71.9 \%)$, and the female sex was 43 people $(28.1 \%)$ with a mean age of 35.27 years and a standard deviation of 12.23 years. The most injured clavicle side was on the right side with a total of 105 people $(68.52 \%)$ and on the left side as many as 48 people (31.48\%). One hundred and forty patients suffered from traffic accidents $(91,5 \%)$. All patients had a right-sided dominant hand (100\%). Based on this descriptive analysis, it was found that the comparison of the number of cases handled conservatively, and operatively was quite different, namely 95 people $(62.1 \%)$ with conservative measures and 58 people (37.9\%) with operative measures. These characteristics are shown in table 1 .

The data obtained is then performed a normality test to determine the distribution of data. The number of samples from the data above was 153 patients so that the normality test was focused on the Shapiro-Wilk column showing a value below $0.05(\mathrm{p}=0.000)$. So that the data is normally distributed for the SF-36 and DASH Score data.

Based on table 2, it can be seen that there is a significant difference between the mean SF36 scores of patients with clavicle fractures of $1 / 3$ of conservative and operative measures. namely 90.81 and $98.21(\mathrm{p}=0.000)$.

Based on table 3, it can be seen that there is a significant difference between the mean DASH score of patients with clavicle fractures of $1 / 3$ of conservative and operative measures. namely 6.94 and $2.42(p=0.000)$. 


\section{QANUN MEDIKA \\ JURNAL KEDOKTERAN FK UM SURABAYA \\ http://journal.um-surabaya.ac.id/index.php/qanunmedika}

Table 1. Characteristics of Patients with Middle 1/3 Clavicle Fracture

\begin{tabular}{lc}
\hline \multicolumn{1}{c}{ Characteristic } & Amount (\%) \\
\hline Gender & $110(71.9 \%)$ \\
Male & $43(28.1 \%)$ \\
Female & \\
Age & $10(6.5 \%)$ \\
$0-18$ & $125(81.7 \%)$ \\
$18-40$ & $18(11.8 \%)$ \\
$40-65$ & \\
Dominant Hand & $153(100 \%)$ \\
Right & $0(0 \%)$ \\
Left & \\
Mode of Injury & $140(91.5 \%)$ \\
Road Traffic Accident & $13(8.5 \%)$ \\
Fall from height & \\
Injury Side & $105(68.62 \%)$ \\
Right & $48(31.38 \%)$ \\
Left & \\
Treatment & $95(62.1 \%)$ \\
Conservative & $80(84.2 \%)$ \\
$\quad$ Armsling & $15(15.8 \%)$ \\
$\quad$ Ransel Verband & $58(37.9 \%)$ \\
Operative &
\end{tabular}

Table 2. Test Results on The SF-36 Based on The Type of Treatment

\begin{tabular}{lllcl}
\hline Treatment & $\mathbf{N}$ & Mean & Standard Deviation & p Score \\
\hline Conservative & 95 & 90.81 & 4.62 & 0.000 \\
Operative & 58, & 98.21 & 1.1 & 0.000 \\
\hline
\end{tabular}

Table 3. Test Results on The DASH Score Based on The Type of Treatment

\begin{tabular}{lllcl}
\hline Treatment & $\mathbf{n}$ & Mean & Standard Deviation & p Score \\
\hline Conservative & 95 & 7.01 & 5.00 & 0.000 \\
Operatives & 58 & 2.54 & 4.16 & 0.000 \\
\hline
\end{tabular}




\section{DISCUSSION}

A clavicle fracture is one of the most common injuries around the shoulder. It has been reported that clavicle fractures account for about $2.6 \%$ of all fractures. Men's incidence is usually highest in the second and third decades, decreasing after that with age. In women, it is generally bimodal, with a peak incidence in the young and the elderly. Allman classified clavicle fractures into three groups based on their location along the bone. Middle third fractures are the most common and account for approximately $80-85 \%$ of clavicle fractures. The narrow cross-section of the bone in the middle trunk combined with the distinctive muscle force acting on it tends to cause fractures at this location. (Kihlström et al., 2017; Naveen et al., 2017).

The goal of treating a clavicle fracture is to reduce pain and make the patient comfortable. There are two treatments for clavicle fracture, namely operative and non-operative. Indications for surgery in clavicle fractures include open fractures, shortening of more than $20 \mathrm{~mm}$, neurovascular problems, puncture of the skin by the fracture fragments. Non-operative management often uses the arm sling or the figure of eight (Virtanen et al., 2012).

This study illustrated that clavicle fractures are quite frequent, with the incidence in Dr. Soetomo Hospital having about 531 cases between December 2019 to January 2020. After exclusion, the final data that match the criteria for this study is 153 patients. The data found that a total of 95 patients $(62.1 \%)$ performed conservative measures and $58(37.9 \%)$ with operative measures. There are different indications for each patient before surgery (surgery or conservative) because we need to see the patient as a whole. Factors that affect can include the patient's background, fracture configuration, the patient's general condition whether surgery can be done or not. Most middle $1 / 3$ clavicle fractures generally heal by any method of immobilization. Therefore, nonoperative treatment is an acceptable modality for clavicle fracture. This is evidenced by the very low rate of nonunion, which was shown by various previous studies. However, recent studies had demonstrated suboptimal outcomes and very high nonunion rates when conservatively managed shear fractures. Other drawbacks of non-operative treatment are functional disorders of the shoulder and noncosmetic lump at the neck's base, which may be due to clavicle shortening and excessive callus formation. Surgical recovery can prevent these drawbacks of conservative treatment. Good outcomes and low complication rates have been reported with various surgical modalities of primary fixation of displaced fractures. However, operative care also has its disadvantages, such as infection of the surgery site, hypertrophic scars, protruding hardware, and repeated surgeries for implant removal at certain times. Because middle 1/3 clavicle fractures generally coalesce with most treatment modalities, clinical trials comparing these therapeutic options are rare. Moreover, there is no uniform consensus on the definite treatment options for displaced middle 1/3 clavicle fractures. (Naveen et al., 2017; Ropars et al., 2017)

The data obtained were predominantly male as many as 110 people $(71.9 \%)$, and female gender, as many as 43 people (28.1\%). This is in accordance with studies in several journals, which suggest that clavicle fractures are more common in patients of the male sex. (Sharma et al., 2018; Tamaoki et al., 2017). Our study found a mean age range of 35.27 years, which indicates that most patients were young adults. (Napora et al., 2016)

Previously, the evaluation of postoperative curative effects was primarily based on neurological dysfunction. In recent years, attention has been paid to patient subjective feelings (assessed using the SF-36 and DASH) 


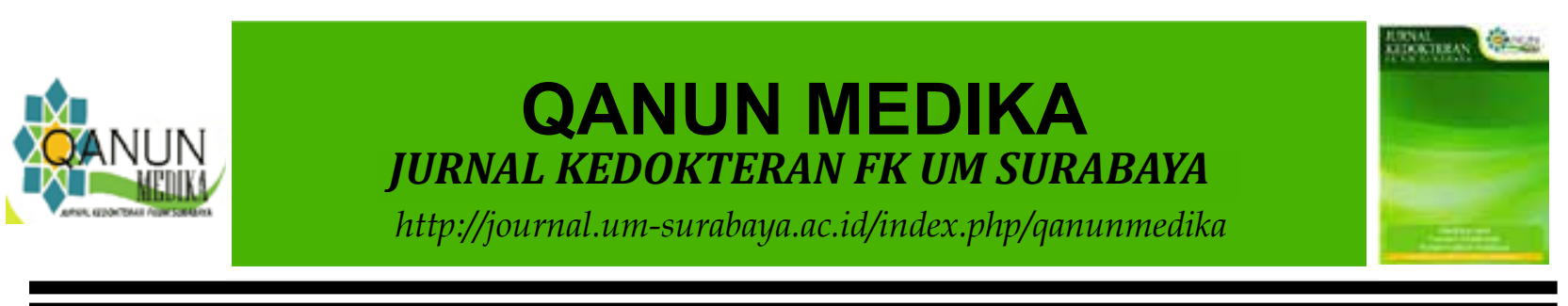

(Zhang et al., 2015). Disability of Arm, Shoulder, and Hand (DASH) questionnaire score is an instrument with specific results that are managed and developed as a measuring tool for disability and upper extremity disorders that we can assess for ourselves. DASH consists of 30 scoring point items, a score of 0 (without disability) to 100 (Gummesson, Atroshi, and Ekdahl, 2003). The Medical Outcomes Study Short-Form Health Survey (SF-36) is a health status questionnaire developed in two decades to assess patients' functional status and health. SF-36 has been used for a variety of clinical purposes, including orthopedics and traumatology. (Zhang, Zhou, and Sun, 2015).

In the evaluation with DASH score, the operative group had significant results with a mean of 2.54 compared to the conservative group with a mean of 7.01. There was also a significant difference between the mean SF36 score of patients with clavicle fractures of $1 / 3$ of conservative and operative measures, namely 90.81 and 98.21 . In the study conducted by Patel, comparing the DASH score in operative patients at the end of the evaluation, the score was 5.16, while in the non-operative group, the result was 6.36. In a randomized controlled trial conducted by Tamaoki et al. in 2017, the difference in DASH scores was not very significant at one-year postoperative evaluation in the operative and conservative groups (Tamaoki et al., 2017). In the Canadian Orthopedic Trauma Society study in 2007, it was found that the DASH score of the nonoperative group was 13, while the DASH score of the operative group was better with a score of 5.2 (Zhang et al., 2015).

The function of the clavicle is as a strut and suspensory. The strut is a bridge between the trunk and the upper extremity. The suspension holds the force from the arm as the place where the upper extremity muscles are attached (Owens \& Goss, 2006). Therefore the clavicle is very influential on the upper extremities.
The AAOS has proposed the DASH score as the standard tool for evaluation of hand and upper limb disability. The value of the DASH score in some common hand conditions was studied (Luc, 2008).

This study shows that the operative action on clavicle fractures shows superiority compared to conservative therapy. Mean SF-36 and DASH scores were better in the surgerytreated group than the conservatively managed group, which was statistically significant. In the past, conservative management was the mainstay of treatment for all clavicle fractures in the middle third, regardless of displacement and comminution, because the clavicle has excellent remodeling power. The conservative treatment with the number 8 bandage acceptably aligns the shifting fragments and yields good functional results (Burnham, Kim, and Kamineni, 2016). However, a recent metaanalysis revealed a higher rate of nonunion for displaced fractures treated non-operatively $(15 \%)$ than surgically (2.2\%) with modern internal fixation techniques. Several recent trials have also revealed a higher incidence of residual pain, nonunion, malunion, shoulder weakness, decreased shoulder endurance, and lower overall satisfaction after non-operative management of middle $1 / 3$ clavicle fracture. (Jarvis et al., 2018).

The existing literature reports two sets of these fracture events: The first is the largest and is associated with an active young population (sports, motor vehicle accidents), while the second is associated with elderly individuals (osteoporotic fractures with simple falls). A direct blow to the shoulder is the most common injury mechanism resulting in a fracture of the middle $1 / 3$ of the clavicle. Since the shoulder is subjected to a compression force from the lateral side, the clavicle and its articulations are the main areas affected when resisting this force. Most (85\%) clavicle fractures occur in the middle due to the narrowest and most 
occasional enveloping soft tissue structure (which can help dissipate the injury force) the rarest. In our study, the age group was 20-50 years. The mean age was 35.2 years. The dominant side was affected in 105 cases $(68.62 \%)$ of 153 subjects. In comparison, the remaining 48 cases $(31.38 \%)$ had fractures on the non-dominant side, similar to the incidence reported in the literature (Ropars, Thomazeau, and Huten, 2017).

Early mobilization in the surgical group helped the patient to maintain initial shoulder strength and function. In contrast, the conservatively treated patient was immobilized for three weeks, resulting in shoulder weakness and delayed shoulder function. Therefore, functional outcomes measured by SF-36 and DASH scores were better in the surgically treated patients at all follow-ups than in the nonsurgical group (Naveen, Joshi, and Harikrishnan, 2017).

Previous studies in adults have shown higher rates of patient satisfaction after non-operative treatment for clavicle fractures. However, patient-reported satisfaction scores may be higher with early surgical stabilization in some circumstances. A multicenter trial reported better functional outcomes, lower malunion and nonunion rates, and shorter overall time to healing in surgically treated clavicle fractures after plate fixation (Naveen, Joshi, and Harikrishnan, 2017; Jarvis et al., 2018).

Previous studies have analyzed the risk of shoulder dysfunction after conservative treatment, commonly associated with shortening bone segments, bone deformity, loss of strength, and persistent pain. Some studies have observed a smaller number of consolidation defects after surgical fixation compared to conservative treatment. In contrast, other studies have shown a $37 \%$ risk of side effects after a surgical procedure may be due to invasion of periosteal structures, which can lead to nerve damage, blood loss, and post-traumatic hematoma, which can delay fracture healing (Ropars, Thomazeau, and Huten, 2017).

Conservative care remains the gold standard in treating simple non-displaced mid-axis clavicle fractures, but surgical intervention is particularly appropriate for displaced fractures and fractures for inactive young adults. If implants and expertise are available, operative treatment can provide satisfactory results and is superior to non-operative care with an excellent surgical technique. Although certain multicenter trials support the use of primary surgical fixation for diaphyseal fractures, this treatment's effect on outcome may not be sufficient to justify surgical treatment for all patients (Burnham et al., 2016).

However, in this study, there is a limitation. There is no more detailed description of the patient receiving operative therapy concerning the methods and tools that might affect the outcome.

\section{CONCLUSION}

This study concludes that surgery is better than conservative treatment in the postoperative functional aspects based on the DASH score and SF-36 score.

\section{REFERENCES}

Burnham, J. M., Kim, D. C., \& Kamineni, S. (2016). Midshaft clavicle fractures: A critical review. Orthopedics, 39(5), e814e821. https://doi.org/10.3928/0147744720160517-06

Carreon, L. Y., Anderson, P. A., McDonough, C. M., Djurasovic, M., \& Glassman, S. D. (2011). Predicting SF-6D utility scores from the neck disability index and numeric rating scales for neck and arm pain. Spine, 36(6), 490. 


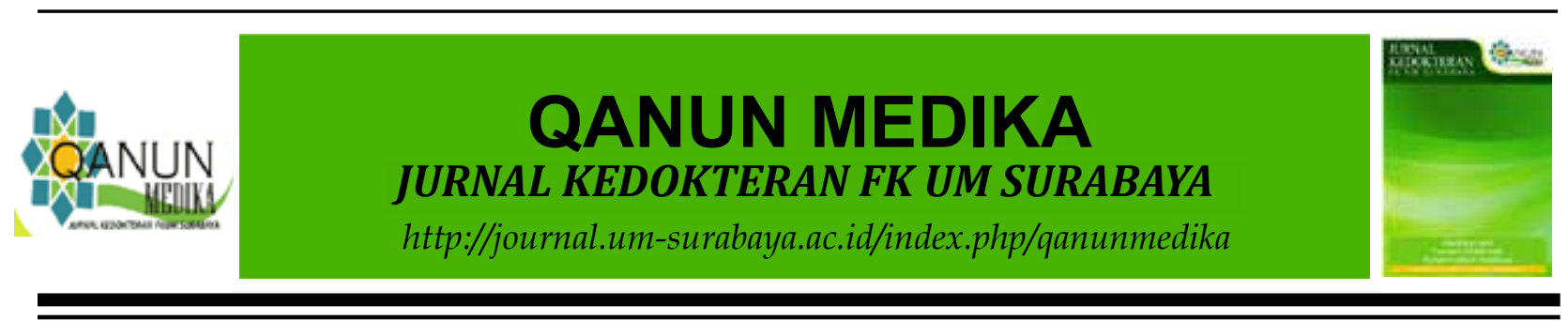

Gummesson, C., Atroshi, I., \& Ekdahl, C. (2003). The disabilities of the arm, shoulder and hand (DASH) outcome questionnaire: Longitudinal construct validity and measuring self-rated health change after surgery. $B M C$ Musculoskeletal Disorders, 4, 1-6. https://doi.org/10.1186/1471-2474-4-11

Jarvis, N. E., Halliday, L., Sinnott, M., Mackenzie, T., Funk, L., \& Monga, P. (2018). Surgery for the fractured clavicle: factors predicting nonunion. Journal of Shoulder and Elbow Surgery, 27(5), e155-e159. https://doi.org/10.1016/j. jse.2017.10.010

Jha, G. K., Timsina, P., Yadav, D., Lamichhane, S., \& Jha, S. (2018). Conservative Vs Operative Management of Displaced Midshaft Clavicle Fracture-A Comparative Study. Biomedical Journal of Scientific \& Technical Research, 11(1), 8293-8303.

Kihlström, C., Möller, M., Lönn, K., \& Wolf, O. (2017). Clavicle fractures: epidemiology, classification and treatment of 2422 fractures in the Swedish Fracture Register; an observational study. $B M C$ Musculoskeletal Disorders, 18(1), 1-9. https://doi.org/10.1186/s12891-0171444-1

Laucis, N. C., Hays, R. D., \& Bhattacharyya, T. (2015). Scoring the SF-36 in orthopaedics: a brief guide. The Journal of Bone and Joint Surgery. American Volume, 97(19), 1628.

Luc, D. (2008). The DASH questionnaire and score in the evaluation of hand and wrist disorders. Acta Orthopaedica Belgica, 74(578), 81-85.
Napora, J. K., Grimberg, D., Childs, B. R., \& Vallier, H. A. (2016). Factors Affecting Functional Outcomes after Clavicle Fracture. Journal of the American Academy of Orthopaedic Surgeons, 24(10), 721-727. https://doi.org/10.5435/ JAAOS-D-16-00130

Naveen, B. M., Joshi, G. R., \& Harikrishnan, B. (2017). Management of mid-shaft clavicular fractures: comparison between non-operative treatment and plate fixation in 60 patients. Strategies in Trauma and Limb Reconstruction, 12(1), 11-18. https://doi.org/10.1007/s11751-0160272-4

Owens, B. D., \& Goss, T. P. (2006). The floating shoulder. The Journal of Bone and Joint Surgery. British Volume, 88(11), 14191424.

Robinson, C. M. (1998). Fractures of the clavicle in the adult: epidemiology and classification. The Journal of Bone and Joint Surgery. British Volume, 80(3), 476484.

Ropars, M., Thomazeau, H., \& Huten, D. (2017). Clavicle fractures. Orthopaedics and Traumatology: Surgery and Research, 103(1), S53-S59. https://doi. org/10.1016/j.otsr.2016.11.007

Sharma, D. S. K., Yadav, D. S. S., Gupta, D. S., Pandey, D. A. P., Ambedkar, D. A., \& Yadav, D. M. S. (2018). Evaluation of functional outcome after plate fixation of midshaft fracture of clavicle. International Journal of Orthopaedics Sciences, 4(3.4), 373-376. https://doi.org/10.22271/ ortho.2018.v4.i3g.64 


\section{QANUN MEDIKA \\ JURNAL KEDOKTERAN FK UM SURABAYA \\ http://journal.um-surabaya.ac.id/index.php/qanunmedika}

Tamaoki, M. J. S., Matsunaga, F. T., Costa, A. R. F. D., Netto, N. A., Matsumoto, M. H., \& Belloti, J. C. (2017). Fractures: Figure-of-Eight Harness Versus Anterior. 1159-1165.

Virtanen, K. J., Malmivaara, A. O. V, Remes, V. M., \& Paavola, M. P. (2012). Operative and non-operative treatment of clavicle fractures in adults: a systematic review of 1,190 patients from the literature. Acta Orthopaedica, 83(1), 65-73.
Zhang, Y., Zhou, F., \& Sun, Y. (2015). Assessment of health-related quality of life using the SF-36 in Chinese cervical spondylotic myelopathy patients after surgery and its consistency with neurological function assessment: A cohort study. Health and Quality of Life Outcomes, 13(1), 1-7. https://doi. org/10.1186/s12955-015-0237-1 